\title{
CARACTERIZACIÓN COGNITIVA Y CONDUCTUAL DE UNA MUESTRA DE ADOLESCENTES CONSUMIDORES DE INHALABLES DE LA CIUDAD DE MÉXICO
}

\section{COGNITIVE AND BEHAVIORAL CHARACTERIZATION OF A SAMPLE OF INHALANTS-CONSUMING ADOLESCENTS FROM MEXICO CITY}

Dailett María Hernández Álvarez¹, Carmen Fernández Cáceres², Jessica Zapata Tellez², Minerva Crespo Ramírez¹ y Nadia González García ${ }^{3}$

\author{
${ }^{1}$ Universidad Nacional Autónoma de México \\ ${ }^{2}$ Centros de Integración Juvenil, México \\ ${ }^{3}$ Hospital Infantil Federico Gómez, México
}

\section{Abstract}

Inhalants are one of the drugs of choice for young people in Mexico City. Its consumption usually begins in a critical period of brain development, associated with processes of myelination and synaptic refinement. There is insufficient information on the cognitive, behavioral and emotional characteristics of inhalants-consuming adolescents. The objective of this study was to determine the neuropsychological profile of the executive functioning of a group of adolescents having Inhalant Use Disorder and to explore the presence of concomitant mental health risk behaviors. Thirty-one adolescents with Inhalant Use Disorder were evaluated through a large test battery for the exploration of executive functioning and a scale on mental health risk behavior. The results show the coexistence of important cognitive and behavioral alterations that are of great relevance for therapeutic treatment of this disorder.

Keywords: Inhalants, adolescence, executive cognitive functioning, mental health.

\section{Resumen}

Los inhalables constituyen una de las drogas de preferencia de los jóvenes de la Ciudad de México. Su consumo inicia generalmente en un período crítico de desarrollo cerebral asociado a procesos de mielinización y refinamiento sináptico. Hay sin embargo, insuficiente información sobre las características cognitivas, conductuales y emocionales de los adolescentes que consumen esta droga. El objetivo de este estudio fue determinar el perfil neuropsicológico del funcionamiento ejecutivo de un grupo de adolescentes con trastorno por consumo de inhalables y explorar la presencia de conductas de riesgo para la salud mental. Treinta y un adolescentes con trastorno por consumo de inhalables se evaluaron mediante una amplia batería de pruebas para la exploración del funcionamiento ejecutivo y una escala sobre conductas de riesgo para la salud mental. Los resultados muestran la coexistencia de alteraciones cognitivas y conductuales de gran relevancia para el manejo terapéutico de este trastorno.

Palabras clave: Inhalables, adolescencia, funciones ejecutivas, salud mental.

Correspondencia: Dra. Nadia García González

nadiag.him@gmail.com 
El consumo de inhalables se identificó en la década de 1960 como un problema de salud en la población marginada o en situación de calle en México. Con los años ha ocurrido una propagación importante en el país de su uso intencional para conseguir efectos psicoactivos y en la actualidad se observa entre personas de todos los estratos socioeconómicos (Medina-Mora et al., 2015).

Estudios epidemiológicos recientes describen que el $71 \%$ de los consumidores de inhalables iniciaron su consumo antes de los 17 años, es decir, durante la adolescencia (Villatoro-Velázquez et al., 2017), período crítico por las transformaciones importantes que acontecen en las estructuras cerebrales (Casey et al., 2005). Los principales cambios en la arquitectura neuronal durante esta etapa de vida ocurren en áreas prefrontales y en la compleja conectividad de éstas con otras regiones cerebrales, asociadas a funciones de regulación y control conductual, cognitivo y emocional (Blakemore \& Choudhury, 2006; Lozano \& Ostrosky, 2011). Se ha sugerido que el principal beneficio de estos procesos de maduración cerebral durante la adolescencia es brindar oportunidad para la conformación de conexiones sinápticas funcionales con base a las necesidades conductuales y ambientales del sujeto (Flores, 2012) y que esta remodelación cerebral si bien permite una mayor eficiencia de la actividad neural y funcionamiento cognitivo, es la que hace a los adolescentes más vulnerables a las adicciones y los desórdenes psiquiátricos (Crews \& Boettinger, 2009; Hackman \& Farah, 2008).

Los inhalables contienen una gran variedad de sustancias químicas, entre sus compuestos orgánicos principales se encuentra el tolueno, compuesto altamente neurotóxico, con efectos sumamente negativos sobre la mielina y la transmisión sináptica. La mayoría de las investigaciones se han realizado en consumidores adultos crónicos y describen síndromes cerebrales difusos, desmielinización, y atrofia asociados a deterioro cognitivo (Rosenberg et al., 2002; Takagi et al., 2008). Algunas investigaciones realizadas en población adolescente muestran que hay presencia de daños en la arquitectura cerebral desde edades tempranas, por ejemplo Aydin et al. (2009) reporta menor volumen en diversas regiones bilaterales de la corteza prefrontal en adolescentes expuestos a altas concentraciones de tolueno respecto a un grupo control y Yücel et al. (2010) describe una menor anisotropía fraccional en las regiones del hipocampo y el esplenio del cuerpo calloso.

El abordaje de la problemática del consumo de inhalables en la población adolescente desde una perspectiva neuropsicológica ha sido pobre a pesar de que algunas investigaciones han informado la presencia de deficiencias significativas en el funcionamiento cognitivo en estos usuarios con respecto a grupos controles y a usuarios de otras drogas (Scott \& Scott, 2013; Takagi et al., 2011). De acuerdo a los datos reportados por dichas investigaciones, los daños cognitivos se caracterizan principalmente por deterioro de la memoria, la atención y una disminución en la velocidad de procesamiento de información. Por otra parte, también son insuficientes los estudios que investigan la coexistencia de alteraciones mentales en la drogodependencia a inhalables en individuos menores de edad, pese a que se ha señalado la existencia de una alta prevalencia de comorbilidad psiquiátrica en la población general de consumidores de esta sustancia (Perron \& Howard, 2009; Wu \& Howard, 2007).

En la presente investigación se tiene como sujetos de estudio una muestra de adolescentes cuyo uso intencional y problemático de inhalables es considerado de acuerdo al Manual Diagnóstico y Estadístico de los Trastornos Mentales DSM-5 como un trastorno por consumo de inhalables. Este trastorno se caracteriza por un deterioro clínico significativo manifestado por un período no menor a doce meses, en el cual el consumo recurrente lleva al individuo a problemas sociales, a la reducción de sus actividades diarias e incluso al riesgo físico (APA, 2013). Las principales directrices que guían esta investigación son determinar el perfil neuropsicológico del funcionamiento ejecutivo de un grupo de adolescentes con trastorno por consumo de inhalables y explorar la presencia de conductas de riesgo para la salud mental concomitante. En este estudio partimos del supuesto, de acuerdo a la literatura científica precedente, que los adolescentes consumidores de inhalables presentan alteraciones significativas en el funcionamiento ejecutivo y una alta tasa de comorbilidad psiquiátrica. Ambos aspectos repercuten significativamente en el proceso de la drogodependencia y en la respuesta del individuo al tratamiento (Robles-Martínez et al., 2017), por lo que una caracterización detallada del estado cognitivo y conductual de esta población puede contribuir al 
desarrollo de estrategias específicas de intervención que eleven la efectividad en los tratamientos.

\section{MÉTODO}

\section{Participantes}

Los adolescentes fueron reclutados por conveniencia en seis Centros de Integración Juvenil (CIJ) para la atención de jóvenes consumidores de drogas distribuidos en la Ciudad de México. Se invitó a participar en el estudio a todos los adolescentes que acudieron a estos CIJ durante el período de mayo de 2016 a Diciembre de 2017 que cumplían con los siguientes criterios de inclusión: edad entre 10 y 17 años de edad, duración del consumo de inhalables igual o mayor a un año, frecuencia de consumo mínima de varias veces a la semana, escolaridad mayor a cuarto grado de primaria y vivir en un entorno familiar. Se excluyeron del estudio a los individuos con diagnóstico de trastorno psicótico actual, discapacidad intelectual, trastorno por consumo de otras sustancias adictivas y antecedentes patológicos prenatales y perinatales.

\section{Instrumentos}

\section{Entrevista estructurada}

La entrevista fue elaborada en función de tres objetivos: a) obtener datos sociodemográficos de interés de los participantes, b) conocer la historia del consumo de drogas: edad de inicio, duración, frecuencia, drogas utilizadas y c) reunir información cualitativa sobre las motivaciones del consumo de los inhalables y percepción del impacto de éste en la salud mental.

\section{Evaluación de las funciones ejecutivas}

La evaluación de las funciones ejecutivas se realizó a través de la Batería de Funciones Ejecutivas y Lóbulos Frontales, BANFE-2 (Flores, Ostrosky-Solís \& Lozano, 2014). Está batería evalúa diversos procesos cognitivos y cuenta con datos normativos en población mexicana de acuerdo a la edad y la escolaridad. A continuación se enlistan las pruebas empleadas y las funciones principales que evalúan.

- Laberintos: Planeación de la conducta visoespacial y respeto de reglas.
- Señalamiento autodirigido: Memoria de trabajo viso-espacial en una tarea autodirigida.

- Ordenamiento alfabético de palabras: Memoria de trabajo verbal.

- Resta consecutiva: Capacidad para desarrollar operaciones mentales consecutivas e inversas.

- Clasificación de cartas: Capacidad para generar una hipótesis de clasificación, cambiar de forma flexible el criterio de clasificación (flexibilidad mental) y mantener una conducta en relación a reforzamiento positivo.

- Generación de clasificaciones semánticas: Capacidad de abstracción y nivel de productividad en relación al número de categorías producidas.

- Efecto Stroop (dos modalidades o tareas): Inhibición de una respuesta automatizada.

- Fluidez verbal: Capacidad de producir fluidamente verbos en un lapso corto de tiempo.

- Juego de cartas: Toma de decisiones que implican evaluación de relaciones de riesgo beneficio.

- Selección de refranes: Abstracción del significado, comprensión del sentido figurado.

- Torre de Hanoi: Planeación secuencial. Implica la realización de pasos intermedios para llegar a una meta.

- Metamemoria: Capacidad para realizar juicios predictivos sobre la capacidad de memoria y ajustes del juicio de acuerdo al desempeño (monitoreo y control).

- Memoria de trabajo viso-espacial: Capacidad de memoria de trabajo viso-espacial para seguir un orden específico.

\section{Evaluación conductual}

Para la descripción de las conductas de riesgo para la salud mental se empleó la traducción y adaptación mexicana (Albores-Gallo et al., 2007) de la versión más reciente del Cuestionario sobre la Conducta Infantil CBCL 
elaborado por Achenbach \& Rescorla (2007) la cual tiene una confiabilidad test-retest de .84 y confiabilidad interna para el total de problemas de.97. Este cuestionario para padres está constituido por 113 ítems que se clasifican en escalas comparables con los criterios diagnósticos del DSM-IV. El cuestionario abarca conductas infantiles asociadas a problemas de ansiedad, depresión, trastorno por déficit de atención e hiperactividad, quejas somáticas, conductas oposicionistas desafiantes y conductas agresivas y delictivas. Cada uno de estos ítems se encuentra configurado con tres opciones de respuesta: falso (0), algunas veces (1), y muy cierto (2).

\section{Procedimiento}

Los adolescentes fueron reclutados por trabajadores profesionales de los CIJ con experiencia en los trastornos de conductas adictivas tomando en cuenta los objetivos del estudio y los criterios de elegibilidad. Posteriormente, los sujetos interesados en compañía de algún familiar pasaron por un proceso de consentimiento donde el entrevistador proporcionaba información más detallada relativa a la confidencialidad del estudio, los riesgos y beneficios, las pruebas psicométricas, la duración del estudio, etc. y recababa información relevante por medio de una entrevista estructurada sobre la historia del consumo de sustancias adictivas. En ese encuentro se les aplicaba la Escala de Inteligencia para Niños, WISC-IV (Wechsler, 2007) o la Escala de Inteligencia para Adultos WAIS-IV (Wechsler, 2013), en dependencia de la edad del sujeto, para descartar la presencia de discapacidad intelectual, mientras que sus familiares respondían preguntas sobre el neurodesarrollo y la Escala sobre Conducta Infantil CBCL. A los voluntarios que cumplían con todos los criterios de investigación, se les administró en una segunda sesión la Batería de Funciones Ejecutivas y Lóbulos Frontales BANFE-2. Todos los procedimientos del estudio fueron llevados a cabo individualmente y se realizaron en un lugar privado y sin distractores proporcionados por las instituciones involucradas. La aplicación de las pruebas se realizó por profesionales de la psicología con experiencia en el uso de éstas. El tiempo promedio de contacto con los participantes fue de una hora y media por sesión. Todos los procedimientos del protocolo fueron aprobados por el Comité de Investigación Científica del Centro de Integración Juvenil. El procesamiento y tratamiento estadístico de los datos se realizó mediante el programa SPSS versión 21.0.

\section{RESULTADOS}

\section{Análisis descriptivo de la muestra}

La muestra quedó constituida por 31 adolescentes con una edad media de 15 años, que incluía 25 hombres y 6 mujeres. Dos individuos fueron excluidos del estudio debido a puntuaciones del cociente intelectual inferiores a 70 que indicaba la presencia de discapacidad intelectual y uno por interrupción voluntaria de la evaluación. El 81\% de los sujetos reportó inhalar más de tres veces a la semana productos rebajadores de pintura, acetona y gasolina y un 19\% reportó hacer uso además de pinturas, desodorantes y pegamentos. La edad media del inicio del consumo fue de $13.02(D T=1.7)$. El tiempo de consumo promedio fue de 24 meses, el tiempo mínimo fue de 12 meses y el máximo de 60 meses.

El $61 \%$ de la muestra reportó consumo de otras drogas aunque en menor frecuencia que los inhalables. En la Tabla 1 se pueden observar las características del policonsumo. El 53.3\% de la muestra reportó cese del consumo de drogas apenas unos días atrás y el $20 \%$ abandono de éstas desde hacía un mes. El 10\% de la muestra reportó no consumir drogas desde hacía tres meses, mientras que el $16.6 \%$ de los participantes mencionó no haber logrado suspender su uso. El 66\% de la muestra describió uno o dos intentos fallidos por abandonar la droga antes de solicitar ayuda profesional.

Tabla 1. Reporte sobre el consumo de drogas

\begin{tabular}{lc}
\hline Solo inhalables & $\begin{array}{c}\text { Número de } \\
\text { sujetos }\end{array}$ \\
Inhalables y alcohol & 5 \\
Inhalables y mariguana & 6 \\
$\begin{array}{l}\text { Inhalables, alcohol y mariguana } \\
\text { Inhalables y cocaína }\end{array}$ & 2 \\
$\begin{array}{l}\text { Inhalables, mariguana y cocaína } \\
\text { Inhalables, alcohol, mariguana y cocaína }\end{array}$ & 2 \\
\hline $\begin{array}{l}\text { Nota: Drogas usadas sistemáticamente (de varias veces por semana a casi } \\
\text { diario). }\end{array}$
\end{tabular}


El nivel educativo promedio de los participantes fue de $8.1(D T=1.1)$ años. Las principales motivaciones descritas que dieron al inicio del consumo de inhalables fueron mitigar malestares emocionales $(63 \%$ de los entrevistados), la mayoría de los cuales se vinculaban con problemas familiares así como fines recreativos (curiosidad y diversión con amigos). A la pregunta ¿a qué problemas le ha llevado su consumo? la respuesta más frecuente fue: a conflictos en el ámbito familiar (63\%) seguida de peleas en la escuela y en la calle (20\%). Los problemas cognitivos autoreportados por los voluntarios fueron: fallos en la memoria en el $77 \%$ de ellos, problemas de atención en el $70 \%$, pobre control de los impulsos en el 64\%, alteraciones en el lenguaje expresivo en el $48 \%$, dificultades en la toma de decisiones en el $48 \%$ y lentitud en el pensamiento también en el $48 \%$. Respecto a su estado emocional el $70 \%$ de los participantes autoreportó sentir ansiedad y el 48\% mencionó sentimientos de depresión.

\section{Análisis del funcionamiento ejecutivo}

La batería neuropsicológica de funciones ejecutivas y lóbulos frontales BANFE-2, permite obtener un perfil individual, que describe las habilidades e inhabilidades del evaluado. Las puntuaciones naturales de las pruebas se transforman en puntuaciones normalizadas con una media de 10 y una desviación estándar de 3. A partir de estos parámetros la prueba permite determinar si el individuo tiene un funcionamiento ejecutivo normal alto, normal, con alteraciones leves a moderadas o con alteraciones severas para cada una de las subpruebas. En la Tabla 2 se muestra por cada subprueba el porcentaje de participantes que puntuó en los cuatro grados de clasificación del funcionamiento ejecutivo y la clasificación de acuerdo a las puntuaciones promedios de la muestra.

De acuerdo a los resultados en la batería BANFE-2 hubo una gran variabilidad en el funcionamiento ejecutivo de los participantes. Los resultados obtenidos mostraron la presencia de adolescentes con puntuaciones dentro del rango de alteraciones leves a moderadas así como dentro del rango de alteraciones severas en todas las pruebas, sin embargo, tomando en cuenta las puntuaciones medias del grupo, el perfil cognitivo tiende a la normalidad en la mayoría de éstas.

Las puntuaciones medias evidencian por otra parte, algunos déficits importantes en el funcionamiento ejecutivo. El bajo rendimiento en la prueba de laberintos, específicamente las dificultades para acatar la regla de no atravesar o tocar las paredes al resolver el ejercicio, pudiera indicar un pobre control de los impulsos. En la tarea de ordenamiento alfabético en el nivel de mayor dificultad (nivel 3), la puntuación media de los participantes se situó dentro del perfil de alteraciones leves a moderadas. Esta tarea está dirigida a evaluar la capacidad de memoria de trabajo verbal y la puntuación media obtenida apunta hacia una pobre retención y dificultad para la manipulación mental de palabras en esta muestra de adolescentes. También se encontraron déficits en los participantes en la capacidad de inhibición de una respuesta automática, pues los puntajes medios en la cantidad de errores Stroop se situaron en el perfil de alteraciones leves a moderadas. Las funciones cognitivas menos afectadas de acuerdo al perfil general de funcionamiento ejecutivo de la muestra fueron la fluidez verbal y la memoria de trabajo visoespacial.

Se realizó un análisis de correlación de Pearson para analizar si la edad de inicio del consumo, el tiempo y la frecuencia del mismo, se relacionan con el rendimiento de los participantes en las tres tareas en las cuales se observó un perfil de desempeño promedio de alteraciones leves a moderadas. Los valores de la $p$ de Pearson se pueden observar en la Tabla 3. No se halló correlación entre las variables del consumo y el número de errores cometidos por atravesar paredes en la prueba de laberintos. Tampoco se halló vínculo entre éstas y los errores de inhibición en la prueba Stroop, pero se observó asociación entre la edad de inicio del consumo de inhalables y la frecuencia del mismo con el desempeño en el nivel máximo de la prueba ordenamiento alfabético. Una menor edad de inicio del consumo y el uso más frecuente de esta droga, se asocia con un menor rendimiento en esta tarea. Estos resultados apuntan a que la frecuencia y la edad de inicio del consumo de inhalables influyen en el desarrollo de la memoria de trabajo verbal.

Al correlacionar de manera cualitativa los datos arrojados por la BANFE-2 con el autoreporte de los participantes sobre los efectos percibidos del consumo de inhalables en el funcionamiento cognitivo, observamos congruencia entre ambos datos, pues como ya habíamos descrito, más del $50 \%$ de los participantes mencionó tener problemas en la memoria y pobre 
control de impulsos lo cual fue confirmado a través de la

evaluación neuropsicológica.

Tabla 2. Perfil neuropsicológico de las funciones ejecutivas

\begin{tabular}{|c|c|c|c|c|c|}
\hline & \multicolumn{4}{|c|}{ Clasificación del funcionamiento ejecutivo } & \multirow[b]{2}{*}{$\begin{array}{c}\text { Clasificación del FE de acuerdo a } \\
\text { la puntuación media de la } \\
\text { muestra }\end{array}$} \\
\hline & $\begin{array}{c}\text { Normal alto } \\
\%\end{array}$ & Normal \% & $\begin{array}{c}\text { Alteración } \\
\text { Leve-Moderada \% }\end{array}$ & Alteración severa \% & \\
\hline \multicolumn{6}{|l|}{ Laberintos } \\
\hline Errores -planeación & 0 & 77 & 10 & 13 & Normal \\
\hline Atravesar bordes & 0 & 42 & 6 & 52 & Alteraciones leve- Moderadas \\
\hline Tiempo de ejecución & 0 & 81 & 13 & 6 & Normal \\
\hline \multicolumn{6}{|l|}{ Señalamiento autodirigido } \\
\hline Aciertos & 9 & 61 & 16 & 14 & Normal \\
\hline Tiempo & 0 & 71 & 9 & 20 & Normal \\
\hline \multicolumn{6}{|l|}{ Ordenamiento alfabético } \\
\hline Nivel 1 & 0 & 52 & 22 & 26 & Normal \\
\hline Nivel 2 & 3 & 64 & 7 & 26 & Normal \\
\hline Nivel 3 & 0 & 22 & 61 & 17 & Alteraciones leve- Moderadas \\
\hline \multicolumn{6}{|l|}{ Resta consecutiva } \\
\hline Aciertos & 0 & 61 & 22 & 17 & Normal \\
\hline Tiempo de ejecución & 0 & 71 & 9 & 20 & Normal \\
\hline \multicolumn{6}{|l|}{ Clasificación de cartas } \\
\hline Aciertos & 0 & 58 & 23 & 19 & Normal \\
\hline Errores de mantenimiento & 0 & 64 & 16 & 20 & Normal \\
\hline \multicolumn{6}{|l|}{ Clasificación semántica } \\
\hline Total de categorías & 0 & 61 & 35 & 4 & Normal \\
\hline Categorías abstractas & 0 & 61 & 20 & 19 & Normal \\
\hline \multicolumn{6}{|l|}{ Stroop A } \\
\hline Errores stroop & 0 & 41 & 9 & 40 & Alteraciones leve- Moderadas \\
\hline Tiempo de ejecución & 7 & 64 & 7 & 22 & Normal \\
\hline \multicolumn{6}{|l|}{ Stroop B } \\
\hline Errores stroop & 0 & 61 & 8 & 21 & Alteraciones leve- Moderadas \\
\hline Tiempo de ejecución & 4 & 61 & 19 & 16 & Normal \\
\hline \multicolumn{6}{|l|}{ Fluidez verbal } \\
\hline Total de palabras & 9 & 66 & 22 & 3 & Normal \\
\hline \multicolumn{6}{|l|}{ Juego de cartas } \\
\hline$\%$ de cartas de riesgo & 16 & 58 & 22 & 4 & Normal \\
\hline Puntuación total & 4 & 58 & 22 & 16 & Normal \\
\hline \multicolumn{6}{|l|}{ Refranes } \\
\hline Aciertos & 5 & 87 & 4 & 4 & Normal \\
\hline \multicolumn{6}{|l|}{ Torre de Hanoi- 1} \\
\hline Total de movimientos & 0 & 84 & 13 & 3 & Normal \\
\hline Tiempo de ejecución & 0 & 74 & 9 & 17 & Normal \\
\hline \multicolumn{6}{|l|}{ Torre de Hanoi 2} \\
\hline Total de movimientos & 0 & 52 & 26 & 22 & Normal \\
\hline Tiempo de ejecución & 0 & 52 & 26 & 22 & normal \\
\hline \multicolumn{6}{|l|}{ Metamemoria } \\
\hline Errores positivos & 0 & 77 & 9 & 14 & Normal \\
\hline Errores negativos & 1 & 71 & 6 & 13 & Normal \\
\hline \multicolumn{6}{|c|}{ Memoria de trabajo visoespacial } \\
\hline \multicolumn{6}{|l|}{ Nivel máximo } \\
\hline & 0 & 64 & 19 & 1 & Normal \\
\hline
\end{tabular}

Nota: FE = Funcionamiento ejecutivo 
Tabla 3. Correlación de Pearson entre variables sobre el consumo de inhalables y variables neuropsicológicas

\begin{tabular}{lccc}
\hline & $\begin{array}{c}\text { Edad de inicio } \\
\text { del consumo }\end{array}$ & $\begin{array}{c}\text { Tiempo de } \\
\text { consumo }\end{array}$ & $\begin{array}{c}\text { Frecuencia } \\
\text { de consumo }\end{array}$ \\
\hline Laberintos & -.12 & -.11 & -.10 \\
Atravesar bordes & & & $-.42 *$ \\
\hline $\begin{array}{l}\text { Ordenamiento } \\
\text { alfabético }\end{array}$ & $.62 * *$ & -.22 & -.09 \\
Nivel 3 & .15 & -.02 & -.03 \\
\hline Stroop A & & -.04 & \\
Errores stroop & -.01 & & \\
\hline Stroop B & & & \\
Errores stroop & & & \\
\hline Nota: * $\mathrm{p}<.05 * * \mathrm{p}<.01$ & & & \\
\hline
\end{tabular}

Nota: * $p<.05, * \star p<.01$

\section{Resultados de la evaluación conductual}

El Cuestionario sobre la Conducta Infantil CBCL (Achenbach, 2001) permite obtener valores numéricos para cada una de las siguientes escalas o subdominios: problemas afectivos, ansiedad, problemas somáticos, trastorno por déficit de atención e hiperactividad, conducta oposicionista desafiante y problemas de conducta. El puntaje obtenido para cada una de las escalas queda ubicado en uno de 3 rangos posibles: menor del percentil 93, entre el percentil 93 y el 97 y mayor al percentil 97. Los datos clínicamente significativos son los dos últimos, entre el percentil 93 y 97, que se considera un rango límite en el cual los sujetos tienen un riesgo medio de presentar los problemas a los que hace referencia la escala en comparación con otros niños/as de su edad. Un percentil superior a 97 indica un riesgo alto de presentar el problema. En la figura 1 se muestra el porcentaje de sujetos en cada rango por cada una de las escalas.



Figura 1. Porcentaje de sujetos en los rangos normal, límite y clínico en cada una de las escalas del CBCL
Al analizar las escalas se observó que más del $50 \%$ de los evaluados presentaban conductas clínicas asociadas a problemas afectivos, ansiedad y problemas de conducta. La puntuación media del grupo en la escala de problemas afectivos fue superior al percentil 97 equivalente a un rango clínico que indica un alto riesgo de padecer tal psicopatología. En el resto de las escalas las puntuaciones se ubicaron entre los percentiles 93 y 97 correspondiente con un rango límite o un riesgo medio de padecer los problemas de salud mental explorados. En ninguna de las escalas se obtuvo un percentil medio inferior a 93 mostrando una gran prevalencia de alteraciones conductuales en la muestra evaluada de adolescentes consumidores de inhalables. Cualitativamente se puede distinguir que la alta prevalencia de problemas afectivos y ansiedad detectada a través de las subescalas correspondientes del cuestionario del $\mathrm{CBCL}$, coincide con el autoreporte de los participantes en la entrevista estructurada quienes en su mayoría mencionaron presentar ansiedad y síntomas depresivos.

Se analizó la relación entre las variables sobre el consumo de inhalables y las escalas del cuestionario CBCL. Se encontró poca relación entre dichas medidas (tabla 4). La edad de inicio, el tiempo y la frecuencia de consumo no mostraron asociación con los problemas afectivos, el Trastorno por déficit de atención e hiperactividad y las conductas oposicionistas desafiantes. Se halló una asociación significativa pero moderada entre la edad de inicio del consumo de inhalables y la ansiedad y el tiempo de consumo y la presencia de problemas somáticos y problemas de la conducta. A menor edad de inicio del consumo de inhalables mayor sintomatología ansiosa y a mayor tiempo de consumo mayor número de problemas somáticos y de conducta.

Tabla 4. Correlación de Pearson entre variables sobre el consumo de inhalables y las escalas del CBCL

\begin{tabular}{lccc}
\hline & $\begin{array}{c}\text { Edad de inicio } \\
\text { del consumo }\end{array}$ & $\begin{array}{c}\text { Tiempo de } \\
\text { consumo }\end{array}$ & $\begin{array}{c}\text { Frecuencia } \\
\text { de consumo }\end{array}$ \\
\hline $\begin{array}{l}\text { Problemas } \\
\text { afectivos } \\
\text { Ansiedad }\end{array}$ & -.11 & .10 & .04 \\
$\begin{array}{l}\text { Problemas } \\
\text { somáticos }\end{array}$ & $-.40^{*}$ & .29 & .27 \\
$\begin{array}{l}\text { Déficit de atención } \\
\text { e hiperactividad }\end{array}$ & -.23 & $.42^{*}$ & .35 \\
$\begin{array}{l}\text { Conducta } \\
\text { oposicionista } \\
\text { desafiante }\end{array}$ & -.01 & .18 & -.03 \\
$\begin{array}{l}\text { Problemas de } \\
\text { conducta }\end{array}$ & -.28 & .30 & .00 \\
\hline Nota: ${ }^{*} p<.05$ & & $.46 *$ & .10 \\
\hline
\end{tabular}




\section{DISCUSIÓN}

El presente estudio estuvo dirigido a la determinación del perfil neuropsicológico del funcionamiento ejecutivo de un grupo de adolescentes con trastorno por consumo de inhalables y la exploración de la presencia concomitante de conductas de riesgo para la salud mental. Nuestros resultados no muestran la presencia de un déficit generalizado en el funcionamiento ejecutivo en los adolescentes y apuntan más bien hacia alteraciones neuropsicológicas específicas. Concretamente se observaron alteraciones en los procesos de control de impulsos, en la capacidad para inhibir una respuesta cognitiva automática y en la memoria de trabajo verbal. Esta última afectada por la edad de inicio del consumo y la frecuencia de uso de la droga. La memoria de trabajo verbal y el control inhibitorio son funciones cognitivas importantes para la toma de decisiones en la vida cotidiana y para el procesamiento e interiorización de los contenidos terapéuticos (Khurana et al, 2017; Wanmaker Yucel et al., 2008). Estos resultados ayudan a comprender los retos del tratamiento de la adicción a inhalables, pues investigaciones con drogodependientes han señalado que dichos déficits en el funcionamiento ejecutivo se asocian con el abandono del proceso terapéutico y aumentan el riesgo a las recaídas (Lorea et al., 2010; Madoz \& Ochoa, 2012).

Pese a que a que el perfil promedio de la muestra apunta hacia déficits cognitivos concretos, hay que destacar que algunos participantes mostraron un perfil más generalizado de deterioro en el funcionamiento ejecutivo. Esta variabilidad en el desempeño neuropsicológico puede deberse a diversos factores como la variabilidad en la historia y patrón de consumo, el período de abstinencia, el consumo de otras drogas además de los inhalables, las diferencias en factores psicosociales como la calidad de la educación e instrucción recibida, etc. cuyo análisis estuvo limitado por el tamaño de la muestra. De manera general nuestros datos señalan la necesidad de una evaluación neuropsicológica como parte del diagnóstico clínico en los adolescentes con trastorno por consumo de inhalables.

El diseño transversal de nuestra investigación es una limitación para conocer si los déficits cognitivos detectados en nuestra muestra existían antes del inicio del consumo. Investigaciones Iongitudinales en adolescentes describen la presencia de una memoria de trabajo débil y un pobre control de impulsos asociados con el uso progresivo de alcohol y mariguana (Khurana et al., 2013; 2017). Las debilidades cognitivas predictoras del consumo progresivo de alcohol y mariguana reportadas por estas investigaciones coinciden con el deterioro ejecutivo encontrado en nuestra muestra de adolescentes consumidores de inhalables, por lo que es probable que los déficits cognitivos detectados en los participantes existieran antes del trastorno. Se necesitan estudios longitudinales que permitan entender si la presencia de déficits en el funcionamiento cognitivo influye en el desarrollo de la conducta adictiva de inhalables durante la adolescencia

El segundo aspecto a destacar en nuestros resultados fue que encontramos una alta presencia de conductas que indican un alto riesgo a padecer trastornos mentales. Más del $50 \%$ de la muestra presenta indicadores conductuales clínicos asociados a trastornos de la afectividad, ansiedad y problemas de conducta y un porcentaje no menos importante mostró indicadores clínicos de trastorno por déficit de atención y trastorno oposicionista desafiante. Si bien nuestro análisis se basa en medidas de tamizaje y no en un diagnóstico clínico psicopatológico, nuestros datos apuntan hacia una elevada frecuencia de alteraciones mentales en los adolescentes con trastorno por consumo de inhalables. Nuestros datos son coherentes con investigaciones previas que reportan gran comorbilidad psiquiátrica en la población general de consumidores de estas sustancias (Martín-Navarrete et al., 2018; Perron \& Howard, 2009; Wu \& Howard, 2007) y llaman la atención hacia la gran presencia de ésta en los consumidores más jóvenes.

Al igual que se discutió la posibilidad de que los déficits en el funcionamiento ejecutivo existiesen antes e influyeran en el desarrollo del trastorno por consumo de inhalables, también queda la duda sobre la contribución de las alteraciones detectadas en la salud mental de los participantes en el inicio y curso del trastorno. La mayoría de los participantes expresó que el inicio del consumo de inhalables estuvo motivado por la necesidad de mitigar malestares emocionales pero no se cuenta con información clínica sobre el estado previo de su salud mental para analizar la posible contribución de este factor. Esperamos que investigaciones futuras 
longitudinales puedan dar respuesta a estos interrogantes.

\section{Conclusiones}

En los adolescentes con trastorno por consumo de inhalables coexisten alteraciones emocionales, conductuales y cognitivas, lo que da muestra de la complejidad de la drogodependencia. Las alteraciones en el funcionamiento ejecutivo y las conductas de riesgo para la salud mental detectadas tienen implicaciones importantes para la prevención, diagnóstico y tratamiento ya que pueden causar la progresión negativa del curso clínico, afectar el mantenimiento de la abstinencia o propiciar la reincidencia en el consumo. La detección y atención temprana de estos aspectos puede ayudar a reducir las implicaciones del consumo a largo plazo, pues la adolescencia brinda una ventana de oportunidad para la recuperación de las funciones dado la gran plasticidad cerebral que la caracteriza. Los resultados obtenidos proporcionan evidencia clara de la necesidad de hacer un diagnóstico individualizado oportuno que incluya no solo factores relacionados con el abuso de la sustancia, sino también del estado cognitivo y la salud mental del adolescente.

\section{Agradecimientos}

Los autores agradecen la financiación recibida por el Consejo Nacional de Ciencia y Tecnología CONACYT (CVU 264242, No de becario 222482, No de apoyo 412003) sin la cual no se hubiera podido llevar a cabo el estudio.

\section{REFERENCIAS}

Achenbach, T., \& Rescorla, L. (2007). Multicultural guide for the ASEBA School-Age form \& profiles. Burlington, VT: University of Vermont. Research Center for Children, Youth and Families.

Albores-Gallo, L., Lara-Munoz, C., Esperon-Vargas, C., Cárdenas, J. A., Pérez, A., \& Villanueva, G. (2007). Validez y fiabilidad del CBCL/6-18. Actas Españolas de Psiquiatría, 35 (6), 393-399.

American Psychiatric Association. (2013). Diagnostic and statistical manual of mental disorders (5th ed.). Arlington, VA: American Psychiatric Publishing.

Aydin, K., Sencer, S., Demir, T., Ogel, K., Tunaci, A., \& Minareci, O. (2002). Cranial MR findings in chronic toluene abuse by inhalation. American Journal of Neuroradiology, 23(7), 1173-1179.
Blakemore, S. J., \& Choudhury, S. (2006). Development of the adolescent brain: Implications for executive function and social cognition. Journal of Child Psychology and Psychiatry and Allied Disciplines, 47 (34), 296-312. doi: 10.1111/j.1469-7610.2006.01611.x

Casey, B. J., Tottenham, N., Liston, C., \& Durston, S. (2005). Imaging the developing brain: what have we learned about cognitive development?, 9(3). doi:10.1016/j.tics.2005.01.011

Crews, F. T., \& Boettiger, C. A. (2009). Impulsivity, frontal lobes and risk for addiction. Pharmacology, Biochemistry and Behavior, 93, 237-247. doi: 10.1016/j.pbb.2009.04.018

Flores, J. (2006). Neuropsicología de los Lóbulos Frontales. México: Universidad Juárez Autónoma de Tabasco, División Académica de Ciencias de la Salud.

Flores, J., Ostrosky, F., \& Lozano, A. (2014). Batería neuropsicológica de funciones ejecutivas y lóbulos frontales BANFE-2. México: El Manual Moderno.

Hackman, D. A., \& Farah, M.J. (2008). Socioeconomic status and the developing brain. Trends in Cognitive Sciences, 13, 65- 73. doi: 10.1016/j.tics.2008.11.003

Khurana, A., Romer, D., Betancourt, LM., Brodsky, NL., Giannetta, JM., Hurt, H. (2013). Working memory ability predicts trajectories of early alcohol use in adolescents: the mediational role of impulsivity. Addiction, 108(3), 506-15. doi: 10.1111/add.12001

Khurana, A., Romer, D., Betancourt, L., \& Hurt, H. (2017). Working Memory Ability and Early Drug Use Progression as Predictors of Adolescent Substance Use Disorders .Addiction, 112(7), 1220-1228. doi: 10.1111/add.13792

Lorea, I., Fernández, J., Tirapu., J., Landa, N., \& López, J. (2010). Rendimiento neuropsicológico en la adicción a la cocaína: una revisión crítica. Revista de Neurología, 51(7), 412- 426.

Lozano, A., \& Ostrosky, F. (2011). Desarrollo de las funciones ejecutivas y la corteza prefrontal. Revista Neuropsicología, Neuropsiquiatría y Neurociencias, 1 (1), 159-172

Madoz, A., \& Ochoa, A. (2012). Alteraciones de funciones cognitivas y ejecutivas en pacientes dependientes de cocaína: estudio de casos y controles. Revista de Neurología, 54(4), 199-208.

Medina-Mora, M., Rafful, C., Villatoro, J.,Oliva, N., Bustos, M., \& Moreno, M. (2015). Diferencias sociodemográficas entre usuarios de otras drogas y adolescentes no consumidores en una muestra mexicana de estudiantes. Revista Internacional de Investigación en Adicciones, 1(1), 6-15. doi: 10.28931/riiad.2015.1.02

Perron, B., \& Howard, M. (2009). Adolescent inhalant use, abuse and dependence. Addiction, 104, 1185-1192. doi:10.1111/j.1360$\underline{0443.2009 .02557 .}$

Robles-Martínez, M., García-Carretero, M., Rahola, G., Palma-Álvarez, R., Abad, A., \& Roncero, Carlos. (2017). Comorbilidad médica y psiquiátrica en pacientes con dependencia alcohólica en tratamiento ambulatorio: un estudio descriptivo. Revista de Patología Dual, 4(3-4), 1-9. doi:10.17579/Revpatdual.04.11 
Rosenberg, N. L., Grigsby, J., Dreisbach, J., Busenbark, D., Rosenberg, N. L., Grigsby, J., et al. (2002). Neuropsychologic Impairment and MRI Abnormalities Associated with Chronic Solvent Journal of Toxicology: Clinical Toxicology, 40(1), 21-34. doi:10.1081/CLT-120002883

Scott, K., \& Scott, A. (2013). Adolescent inhalant use and executive cognitive functioning. Child: Care, Health, and Development, 40(1), 2028. doi:10.1111/cch.12052

Takagi, M. J., Lubman, D. I., \& Yücel, M. (2008). Interpreting neuropsychological impairment among adolescent inhalant users: Two case reports. Acta Neuropsychiatrica, 20(1), 41-43. doi:10.1111/j.1601-5215.2007.00236

Takagi, M., Yücel, M., Cotton, SM., \& Baliz, Y. (2011). Verbal memory, learning, and executive functioning among adolescent inhalant and cannabis users. Journal of Studies on Alcohol and Drugs, 72(1), 96-105.

Villatoro-Velázquez JA., Reséndiz-Escobar, E., Mujica-Salazar, A., BretónCirett, M., Gutiérrez-Reyes, J., Franco-Núñez, A., ... MendozaAlvarado, L. (2017). Encuesta Nacional de Consumo de Drogas, Alcohol y Tabaco 2016-2017: Reporte de Drogas. México: Instituto Nacional de Psiquiatría Ramón de la Fuente Muñiz

Wanmaker, W., Jorinde S., Geraerts, E., van de Wetering, B., Renkema, P., \& Franken, I. (2018). The efficacy of a working memory training in substance use patients: A randomized double-blind placebocontrolled clinical trial.Journal of Clinical and Experimental Neuropsychology, 40(5), 473-486.

doi:10.1080/13803395.2017.1372367

Wechsler, D. (2007). Escala Wechsler de inteligencia para niños WISC- IV. México: El Manual Moderno.

Wechsler, D. (2013). Escala Wechsler de inteligencia para adultos WAIS- IV. México: El Manual Moderno.

Wu, L., \& Howard, M. (2007). Psychiatric disorders in inhalant users: Results from The National Epidemiologic Survey on Alcohol and Related Conditions. Drug \& Alcohol Dependence, 88(2) 146 - 155. doi:10.1016/j.drugalcdep.2006.10.012

Yücel, M., Zalesky, A., Takagi, MJ., Bora, E., Fornito, A., Ditchfield, M., ... Lubman, Ph. (2010). White-matter abnormalities in adolescents whit long-term inhalant and cannabis use: a diffusion magnetic resonance imaging study. Journal of Psychiatry \& Neuroscience, 35(6), 409-12. doi: 10.1503/jpn.090177 